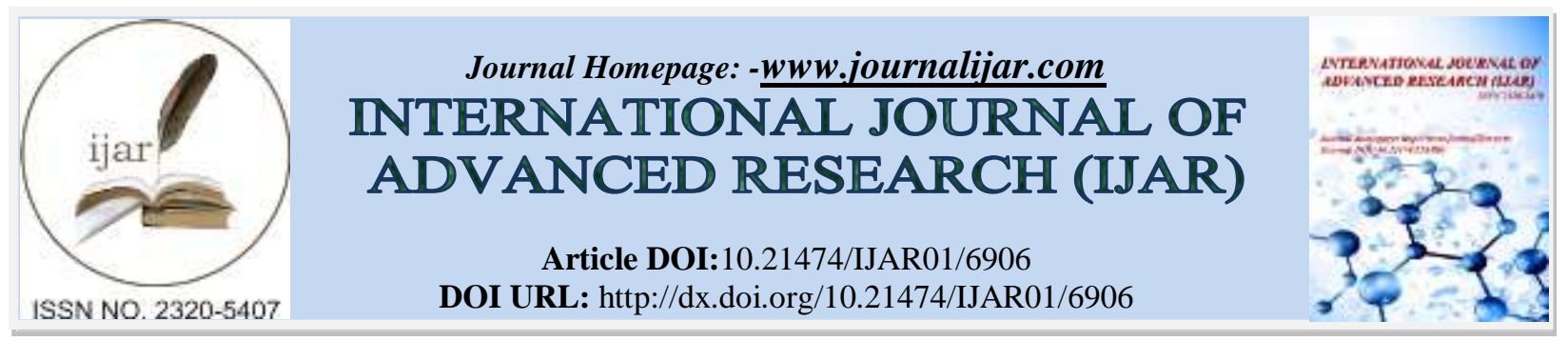

RESEARCH ARTICLE

\title{
MATHEMATICAL ANALYSIS OF MULTICOMPARTMENT EPIDEMIC MODEL.
}

\section{Laid chahrazed.}

Department of Mathematics, Faculty of Exact Sciences , University Freres Mentouri, Constantine, Algeria.

\section{Manuscript Info}

Manuscript History

Received: 11 February 2018

Final Accepted: 13 March 2018

Published: April 2018

Keywords:-

Basic reproduction number, endemic equilibrium, Local asymptotic stability, stochastic stability.

\section{Abstract}

In this paper, we study a nonlinear mathematical model in population with variable size. Size $\mathrm{N}(\mathrm{t})$ at time $\mathrm{t}$, is divided into eight sub classes, with $\mathrm{N}(\mathrm{t})=\mathrm{S}(\mathrm{t})+\mathrm{I}(\mathrm{t})+\mathrm{I}_{1}(\mathrm{t})+\mathrm{I}_{2}(\mathrm{t})+\mathrm{I}_{3}(\mathrm{t})+\mathrm{I}_{4}(\mathrm{t})+\mathrm{Q}(\mathrm{t})+\mathrm{R}(\mathrm{t})$; where $\mathrm{S}(\mathrm{t})$, $\mathrm{I}(\mathrm{t})$, and $\mathrm{Q}(\mathrm{t})$ denote the sizes of the population susceptible to disease, and infectious members, quarantine members with the possibility of infection through temporary immunity, respectively. The stability of a disease-free status equilibrium and the existence of endemic equilibrium can be determined by the ratio called the basic reproductive number. This paper study the equilibrium, local stability and and the stochastic stability of the free disease equilibrium under certain conditions .

\section{Introduction:-}

This paper considers the following epidemic model with temporary immunity:

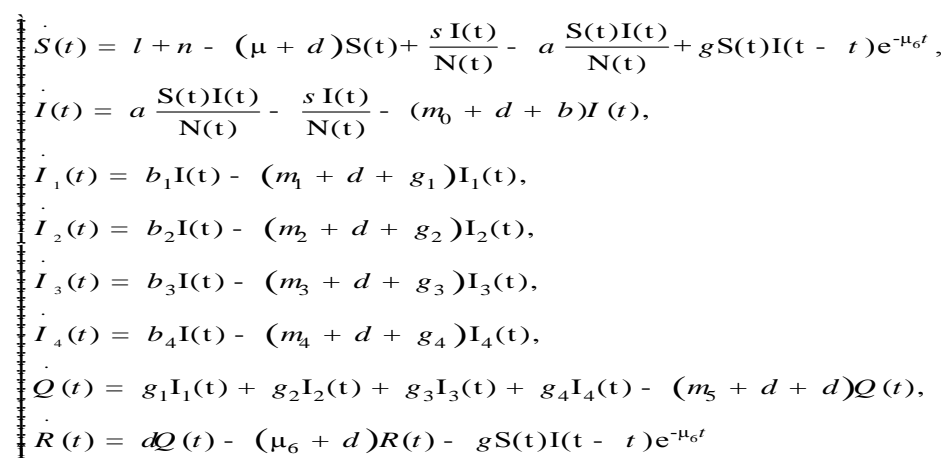

Consider a population of size $\mathrm{N}(\mathrm{t})$ at time $\mathrm{t}$, this population is divided into for sub-classes, with $\mathrm{N}(\mathrm{t})=\mathrm{S}(\mathrm{t})+\mathrm{I}(\mathrm{t})$ $+\mathrm{I}_{1}(\mathrm{t})+\mathrm{I}_{2}(\mathrm{t})+\mathrm{I}_{3}(\mathrm{t})+\mathrm{I}_{4}(\mathrm{t})+\mathrm{Q}(\mathrm{t})+\mathrm{R}(\mathrm{t})$.

Where $S(t), I(t), I_{1}(t), I_{2}(t), I_{3}(t), I_{4}(t), Q(t)$ and $R(t)$ denote the sizes of the population susceptible to disease, infectious members, quarantine members with the possibility of infection through temporary immunity, and who were removed from the possibility of infection respectively. The positive constants $\mu_{1}, \mu_{2}, \mu_{3}, \mu 4, \mu 5$ and $\mu 6$ represent the death rates of susceptible, infectious, quarantine and removed. Biologically, It is natural to assume that $\mu \leq \min \left\{\mu_{0}, \mu_{1}, \mu_{2}, \mu_{3}, \mu_{4}, \mu_{5}, \mu_{6}\right\}$. 
The positive constant $d$ is natural mortality rate. The positive constant $\beta=\beta 1+\beta 2+\beta 3+\beta 4$ is the average numbers of contacts. The positive constants $\lambda$ represent the incidence rate of the population. The positive constants $\gamma_{1}, \gamma_{2}, \gamma_{3}$, and $\gamma_{4}$ is the numbers of transfer or conversion of infected people quarantined. $d$ the number of transfer or conversion of Q to R. $v$ the positive constant is the parameter of immigration. $\alpha$ the positive constant is the parameter of emigration The term $g S(t) \mathrm{I}(\mathrm{t}-t) \mathrm{e}^{-\mu_{3} t}$ indicates that an individual has quarantined in a pool recovery before becoming susceptible again, where $\tau$ is the length of immunity period.

The initial condition of (1) is given as:

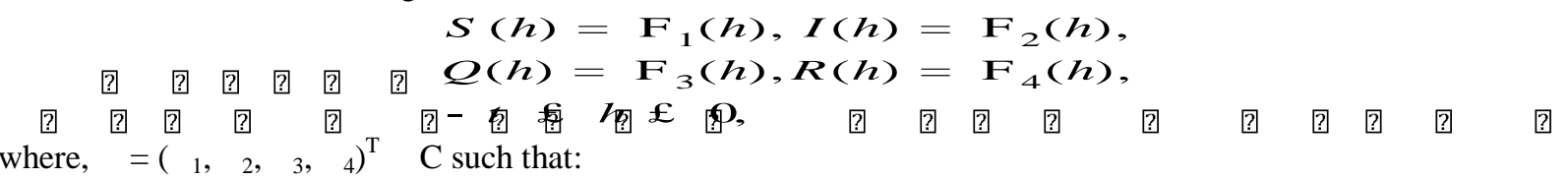

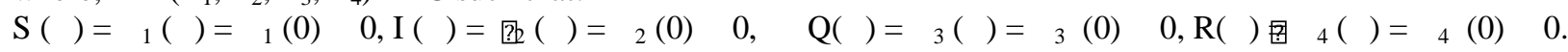

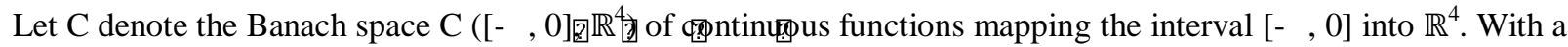
biological meaning, we further assume that:

$$
i()=\quad(0) \quad 0 \text { for } i=1,2,3,4 \text {. }
$$

With the initial condition in (2) which becomes:-

$$
\begin{aligned}
& S(h)=F_{1}(h), I(h)=F_{2}(h), \\
& Q(h)=F_{3}(h), R(h)=F_{4}(h), \\
& -t \notin h \pm O .
\end{aligned}
$$

$\begin{array}{lllllll} & 0 & 0 & 0 & 0\end{array}$

Where,

${ }_{1}(0) \quad 0, \quad 2(0) \quad 0, \quad 3(0) \quad 0, \quad 4(0) \quad 0,-\quad 0$.

The region $\begin{aligned} & \mathrm{W}\left(S(t), I(t), I_{1}(t), I_{2}(t), I_{3}(t), I_{4}(t), Q(t), R(t)\right) \hat{I} i_{i}^{8}, \\ & \mathrm{H}\end{aligned}$

Hence system (1) can be rewritten as

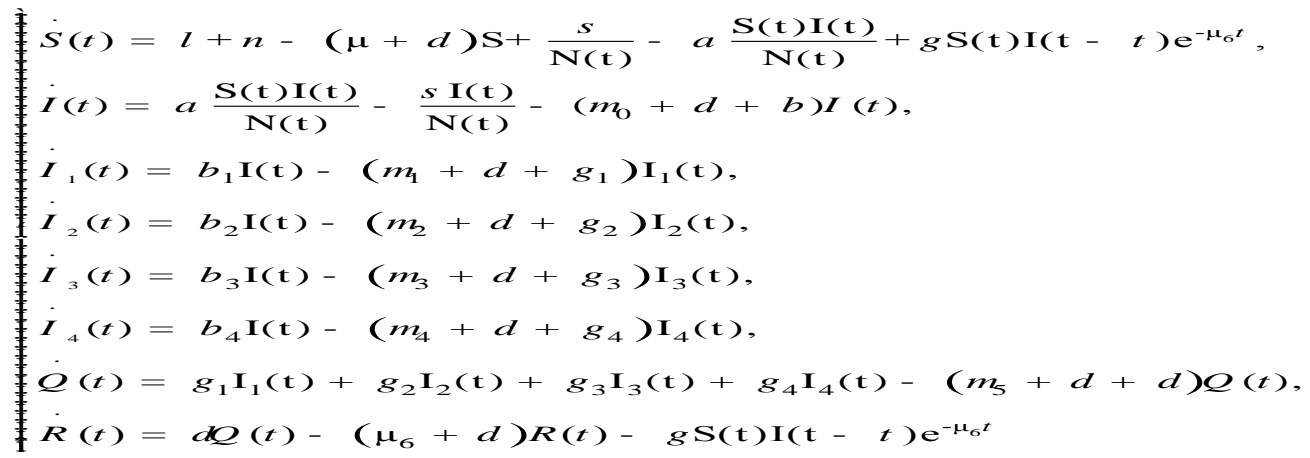

\section{Equilibrium Points:-}




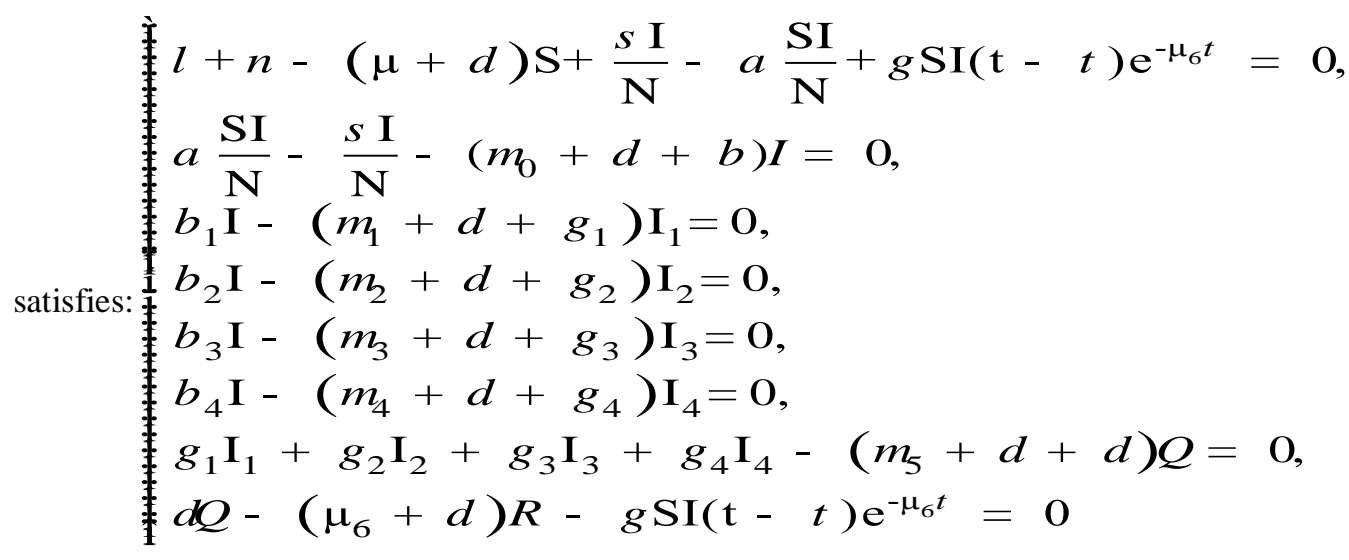

We calculate the points of equilibrium in the absence and presence of infection. In the absence of infection, the system (5) has a disease-free equilibrium $\mathrm{E}_{0}$ :

$$
\mathrm{E}_{0}=\left(\boldsymbol{s}, \$, \boldsymbol{\mu}_{1}, \boldsymbol{\mu}_{2}, \boldsymbol{\mu}_{3}, \boldsymbol{H}_{4}, \boldsymbol{Q}^{\boldsymbol{L}}, \boldsymbol{k}^{\boldsymbol{l}}\right)^{T}=\left(\frac{l+n-a}{m+d}, 0,0,0,0,0,0,0\right)^{T} \text {. }
$$

The eigenvalues can be determined by solving the characteristic equation of the linearization of (4) near $E_{0}$. So, the eigenvalues are:

$$
\begin{aligned}
& A_{1}=-(\mu+\mathrm{d}), A_{2}=a-\frac{s}{N^{*}}-\left(\mu_{0}+d+b\right), \\
& A_{3}=-\left(\mu_{1}+\mathrm{d}+g_{1}\right), A_{4}=-\left(\mu_{2}+d+g_{2}\right) \\
& A_{5}=-\left(\mu_{3}+d+g_{3}\right), A_{6}=-\left(\mu_{4}+d+g_{4}\right) \\
& A_{7}=-\left(\mu_{6}+d+g_{6}\right), A_{8}=-\left(\mu_{6}+d\right)
\end{aligned}
$$

In order to $A_{2}$, will be negative, then we define the basic reproduction number of the infection $R_{0}$ as follows:

$$
R_{0}=\frac{a(l+n)}{s(m+d)+(l+n)\left(m_{0}+d+b\right)}
$$

In the presence of infection, substituting in the system, $\Omega$ also contains a unique positive, endemic equilibrium

$$
\begin{aligned}
& E_{t}^{*}=\left(S_{t}^{*}, I_{t}^{*}, I_{1 t}^{*}, I_{2 t}^{*}, I_{3 t}^{*}, I_{4 t}^{*}, Q_{t}^{*}, R_{t}^{*}\right)^{T} \\
& E_{t}^{*}=\left(S_{t}^{*}, I_{i t}^{*}, Q_{t}^{*}, R_{t}^{*}\right)^{T}, " \mathrm{i}=1,2,3,4 .
\end{aligned}
$$

Where:-

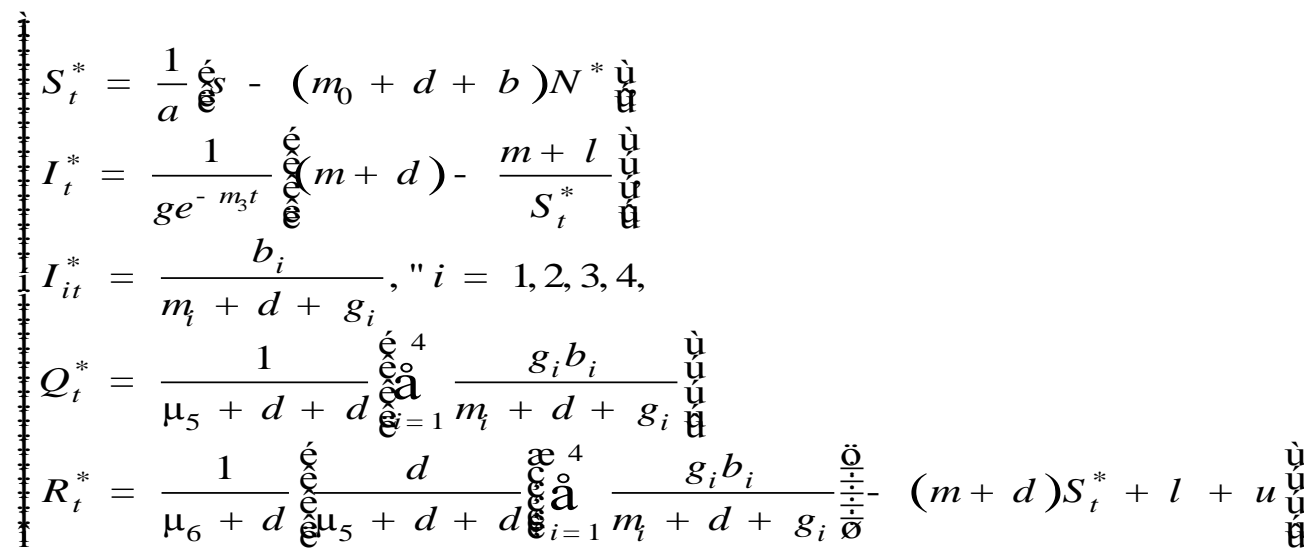




\section{Proposition}

Let $\left(S, I, I_{1}, I_{2}, I_{3}, I_{4}, Q, R\right)$, the solution of the system (4) is defined in $(0, \infty]$ and

$$
\operatorname{Lim}_{t \rightarrow \infty} \sup N(t) \leq \frac{\mu+\lambda}{\mu+d}
$$

\section{Proof}

We have $\dot{N}=v+\lambda-\mu S-\mu_{0} I-\mu_{1} I_{1}-\mu_{2} I_{2}-\mu_{3} I_{3}-\mu_{4} I_{4}-\mu_{5} Q-\mu_{6} R-d N$,

$$
\dot{N} \leq v+\lambda-(\mu+d)
$$

By integration,

$$
\begin{aligned}
& N(t) \leq \frac{v+\lambda}{\mu+d}\left(1-e^{-(\mu+d) t}\right), \text { for every } \quad t \in(0, T], \\
& N(t) \leq 2 . \frac{\mu+\lambda}{\mu+d}
\end{aligned}
$$

The solutions of sub-populations are bounded in the interval $(O, T]$.

Then we have $N(t) \leq \frac{v+\lambda}{\mu+d}\left(1-e^{-(\mu+d) t}\right)$, for every $t \in(0, \infty]$

Finally $\operatorname{Lim}_{t \rightarrow \infty} \sup N(t) \leq \frac{\mu+\lambda}{\mu+d}$

\section{The local stability of the free-disease equilibrium :-}

\section{Theorem 1:-}

The disease-free equilibrium $E_{o}$ is locally asymptotically stable if and only if

Proof:- Let

$$
\alpha<\frac{\sigma(v+\lambda)}{\mu+d}-\left(\mu_{0}+d+\beta\right)
$$

$$
\begin{aligned}
& x=S-\frac{v+\lambda}{\mu+d}, y=I, y_{i}=I_{i}, i=1,2,3,4, \\
& z=Q, u=R, w=N-\frac{v+\lambda}{\mu+d} .
\end{aligned}
$$

With the changement, the system (4) becomes

$$
\begin{aligned}
& \dot{x}=[-(\mu+d)] x+\left[\frac{\sigma}{w+N}+\left(\gamma e^{-\mu_{6} \tau}-\frac{\alpha(\mu+d)}{w(\mu+d)+(v+\lambda)}\right) S\right] y, \\
& \dot{y}=\left[\frac{\alpha S-\sigma}{w+N}-\left(\mu_{0}+d+\beta\right)\right] y, \\
& \dot{y}_{i}=\beta_{i} y-\left(\mu_{i}+d+\gamma_{i}\right) y_{i}, \forall i=1,2,3,4, \\
& \dot{z}=\gamma_{1} y_{1}+\gamma_{2} y_{2}+\gamma_{3} y_{3}+\gamma_{4} y_{4}-\left(\mu_{5}+d+\delta\right) z, \\
& \dot{u}=\left[-\gamma e^{-\mu_{6} \tau} S\right] y+\delta z-\left(\mu_{6}+d\right) u .
\end{aligned}
$$


With the linearized of system (7) at the point $(0,0,0,0,0,0,0,0)$, we obtain the eigenvalues,

$$
\begin{aligned}
& A_{i}=-\left(\mu_{i}+d+\gamma_{i}\right), \forall i=1,2,3,4 \\
& A_{5}=-(\mu+d) \\
& A_{6}=\alpha-\frac{\sigma}{N}-\left(\mu_{0}+d+\beta\right) \\
& A_{7}=-\left(\mu_{5}+d+\delta\right) \\
& A_{8}=-\left(\mu_{6}+d\right)
\end{aligned}
$$

The eigenvalues have a negative real part, so;

$E_{o}$ is locally asymptotically stable if and only if $\alpha<\frac{\sigma(v+\lambda)}{\mu+d}-\left(\mu_{0}+d+\beta\right)$.

\section{Stochastic stability of the free-disease equilibrium:-}

We limit ourselves here to perturbing only the contact rate so we replace $k$ by $a+a b(t)$, where $b(t)$ is white noise (Brownian motion). The system (4) is transformed to the following Itô stochastic differential equations:

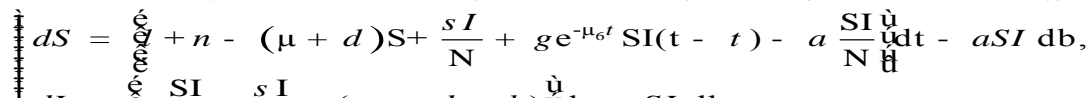

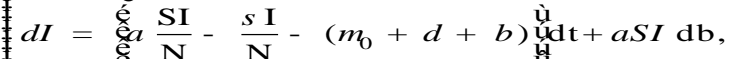

$$
\begin{aligned}
& d I_{1}=e_{e_{1}} \mathbf{I}-\left(m_{1}+d+g_{1}\right) I_{1} \text { ùd } \mathrm{ut} \text {, } \\
& \frac{1}{d I_{2}}=e_{2} b_{2} \mathbf{I}-\left(m_{2}+d+g_{2}\right) \mathbf{I}_{2} \text { ùd t } \text {, } \\
& d I_{3}=\dot{g}_{3} I-\left(m_{3}+d+g_{3}\right) I_{3} \text { ùd } \mathbf{u} \text {, } \\
& d I_{4}=\oint^{2} I_{-}-\left(m_{4}+d+g_{4}\right) \mathbf{I}_{4} \text { ùddt, } \\
& d Q=e^{2} g_{1} \mathbf{I}_{1}+g_{2} \mathbf{I}_{2}+g_{3} \mathbf{I}_{3}+g_{4} \mathbf{I}_{4}-\left(m_{5}+d+d\right) Q \text { ùd } \mathrm{dt} \text {, }
\end{aligned}
$$

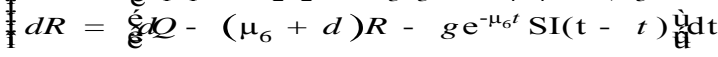

Theorem 2:- If $R_{O}<1, I(t)$ and $R(t)$ are exponentially almost surely stable.

Proof:-

Let $w$ such that $\frac{\sigma(\mu+d)}{\lambda+v}+\left(\mu_{0}+d+\beta\right)-\left(\frac{\alpha(\mu+d)}{\lambda+v}-w \gamma \mathrm{e}^{-\mu_{6} \tau}\right)\left(\frac{\lambda+v}{\mu+d}\right)>0$

Withe Itô's formula, we obtain

$d \log (I+w R)=\frac{1}{I+w R}\left[\begin{array}{l}\left(\frac{\alpha}{N}-w \gamma \mathrm{e}^{-\mu_{6} \tau}\right) \mathrm{SI} \\ -\left(\frac{\sigma}{N}+\left(\mu_{0}+d+\beta\right)\right) I+w \delta Q \\ -w\left(\mu_{6}+d\right) R-\frac{a^{2}(\mathrm{SI})^{2}}{2(I+w R)}\end{array}\right] d t+\frac{a S I}{I+w R} d b$
$d \log (I+w R) \leq \frac{-1}{(I+w R)}\left[\begin{array}{l}\left.\left[\begin{array}{l}(\lambda+v) w \gamma \mathrm{e}^{-\mu_{6} \tau} \\ \mu+d\end{array}\right)-\alpha+\frac{\sigma(\mu+d)}{\lambda+v}\right] I \\ +\left(\mu_{0}+d+\beta\right) \\ +\left(w\left(\mu_{6}+d\right)\right) R\end{array}\right] d t+\frac{a S I}{I+w R} d b$ 
We suppose that

$$
M=\min \left\{\left(\left(\frac{(\lambda+v) w \gamma \mathrm{e}^{-\mu_{6} \tau}}{\mu+d}\right)-\alpha+\frac{\sigma(\mu+d)}{\lambda+v}+\left(\mu_{0}+d+\beta\right)\right), w\left(\mu_{6}+d\right)\right\}
$$

Then

$$
d \log (I+w R) \leq-M d t+\frac{a S I}{I+w R} d b
$$

With integration, we obtain

$$
\log (I+w R) \leq-M d t+a \int_{0}^{t} \frac{S(v) I(v)}{(I(v)+w R(v))} d b(v) \cdot
$$

We have

$\left(\frac{S(v) I(v)}{(I(v)+w R(v))}\right)^{2}$ is bounded. Then

$\operatorname{Lim}_{t \rightarrow \infty} \int_{0}^{t} \frac{S(v) I(v)}{(I(v)+w R(v))} d b(v)=0$ almost surely.

The following form from Doob's martingale inequality combined with Itô isometry see [17].

$$
\operatorname{Lim}_{t \rightarrow \infty} \sup \frac{1}{t} \log (I+w R) \leq-M \quad \text { almost surely }
$$

Then we have

$$
\begin{aligned}
& \operatorname{Lim}_{t \rightarrow \infty} \sup \frac{1}{t} \log I \leq-M \\
& \operatorname{Lim}_{t \rightarrow \infty} \sup \frac{1}{t} \log R \leq-M
\end{aligned}
$$

\section{Theorem 3.}

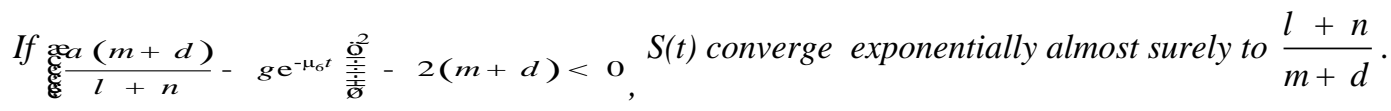

Proof:

Applying Itô formula to the first equation in system (8), we obtain

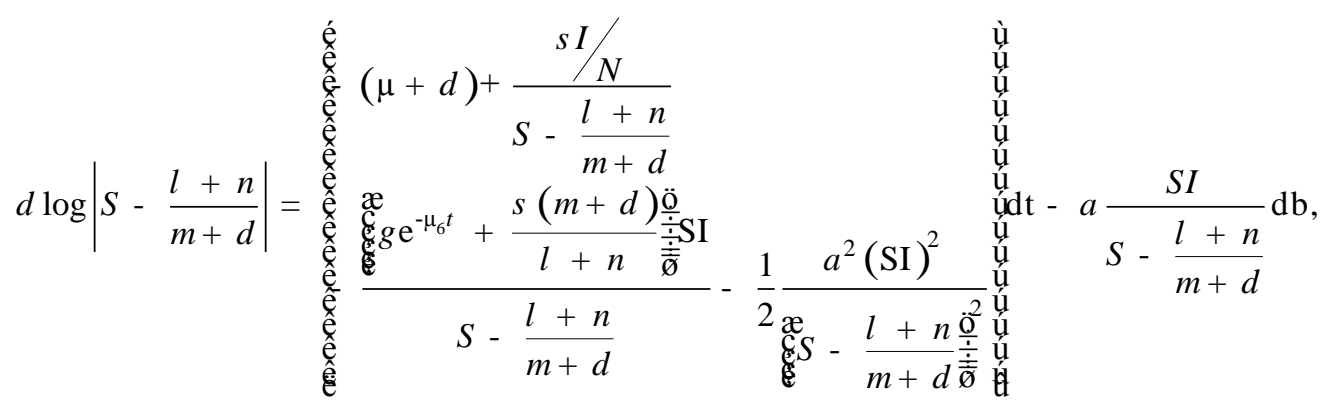




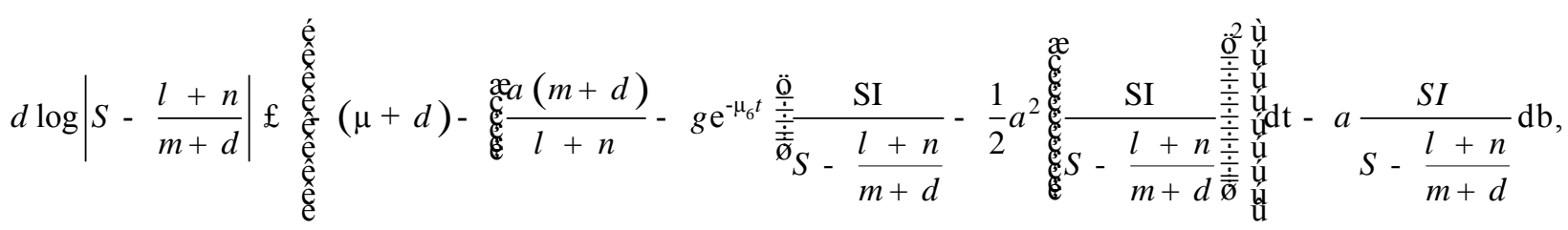

We suppose that

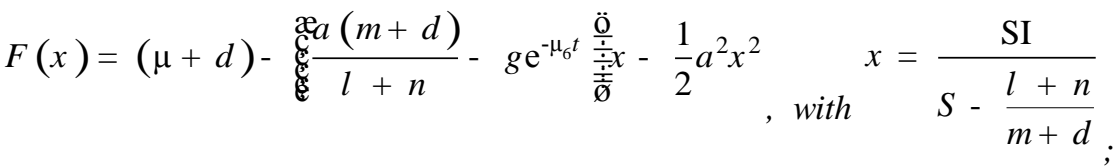

$$
\begin{aligned}
& d \log \left|S-\frac{l+n}{m+d}\right|=F(x) \mathrm{dt}-a \frac{S I}{S-\frac{l+n}{m+d}} \mathrm{db},
\end{aligned}
$$

If the determinant of the equation is negative, then for all $x$.

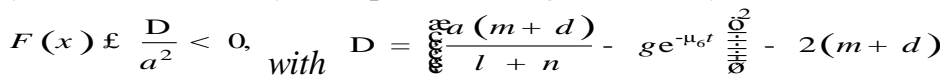

We have

$$
d \log \left|S-\frac{l+n}{m+d}\right| £ \frac{\mathrm{D}}{a^{2}} \mathrm{dt}-a \frac{S I}{S-\frac{l+n}{m+d}} \mathrm{db},
$$

With integration, we obtain

$$
\log \left|S-\frac{l+n}{m+d}\right| £ \frac{\mathrm{D}}{a^{2}} \mathrm{t}-a_{0}^{t} \frac{S(v) I(v)}{S(v)-\frac{l+n}{m+d}} d b(v)
$$

Since

$$
\begin{aligned}
& \operatorname{Lim}_{t \circledast 7} \frac{1}{t} \underset{0}{\mathrm{O}} \frac{S(v) I(v)}{S(v)-\frac{l+n}{m+d}} d b(v)=0 \text { almost surely } \\
& \operatorname{Lim}_{t \circledast 7} \sup \frac{1}{t} \log \left|S-\frac{l+n}{m+d}\right| £ \frac{\mathrm{D}}{a^{2}} .
\end{aligned}
$$

\section{Conclusion:-}

In this paper, the epidemic model has a disease free equilibrium $\mathrm{E}_{0}$, witch is locally asymptotically stable if and only if $\alpha<\frac{\sigma(v+\lambda)}{\mu+d}-\left(\mu_{0}+d+\beta\right)$ and the endemic equilibrium $E_{t}^{*}$.

We proof $I(t)$ and $R(t)$ are exponentially almost surely stable if If $R_{0}<1$, Finally $S(t)$ converge exponentially almost

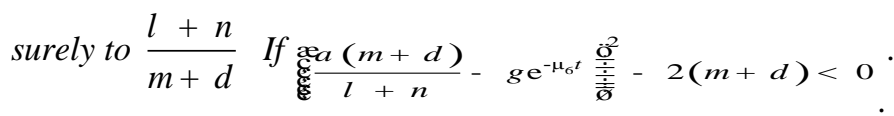




\section{References:-}

1. A. Lahrouz, L. Omari, D. Kiouach. Global analysis of a deterministic and stochastic nonlinear SIRS epidemic model.Nonlinear Analysis: Modelling and Control,Vol. 16, No. 1, 2011, pp 59-76.

2. Anderson R. M and Medley R M and Johnson. A. K. A Preliminary Study of the Transmission Dynamics of the Human Immunodeficiency Virus (HIV), the Causative Agent of AIDS. IMA. J. Math. Appl. Med. Biol 3, 1986, pp. 229-263.

3. Abta A and Kaddar A and Talibi H. A. Global Stability for Delay SIR and SEIR Epidemic Models with Saturated Incidence Rates. Electronic Journal of Differential Equations, 23, 2012, pp. 1-13.

4. B. Bonzi, A. A. Fall, Abderrahman Iggidr, Gauthier Sallet. Stability of differential susceptibility and infectivity epidemic models.Journal of Mathematical Biology, Springer Verlag (Germany), 2011, 62 (1), pp.39-64

5. Bailley. N.T.J. Some Stochastic Models for Small Epidemics in Large Population. Appl. Statist.13, 1964, pp. 919.

6. Bailley. N.T.J. The Mathematical Theory of Infection Diseases and its Application. Applied Statistics, 26, N1, 1977, pp. 85-87.

7. Batiha, M. S. M. Noorani and I. Hashim. Numerical solutions of the nonlinear integro-differential equations, Int. J. Open Probl. Compt. Math, 2008, pp. 34-42.

8. Becker.N.G. The Uses of Epidemic Models. Biometrics 35, 1979 pp. 295-305.

9. Billard.L. A Stochastic General Epidemic in m Sub-Population. J. Appl. Prob. 13, 1976, pp. 567-572.

10. James M. Hyman, Jia Li. The reproductive number for an HIV model with differential infectivity and staged progression.

11. Linear Algebra and its Applications 398. 2005, pp 101-116.

12. James M. Hyman. Jia Li. Epidemic Models with differentialsusceptibility and staged progression and their dynamics. Mathematical Biosciences and engineering. Volume 6, Number 2 , April 2009, pp . 321-332.

13. Jinliang W, Xinxin Tian. Global Stabilty of a Delay Differential Equation of Hepatitis B Virus Infection with Immune Response. Electronic Journal of Differential Equations, 2013, 94, pp. 1-11.

14. Jin. Z, Zhien. M and Maoan. H. Globale stability of an SIRS epidemic model with delay. Acta Matimatica Scientia. 26 B, 2006, pp. 291-306.

15. Kuang Y. Delay-Differential Equations with application in population biology. Academic Press, new york. 1993.

16. Lounes. R and Arazoza. H. Modeling HIV Epidemic Under Contact Tracing. The Cuban Case. Journal of theoritical Medecine Vol 2, 2000, pp. 267-274.

17. Lounes. R, Arazoza. H. A Non-Linear Model for a Sexually Transmitted Disease with contact tracing. IMA. J. MJath. Appl. Med. Biol.19, 2002, pp. 221-234.

18. Lahrouz A and El Maroufy H. Qualitative Behaviour of a Model of an SIRS Epidemic Stability and Permanence. Applied Mathematics \& Information Sciences. An International Journal 5 (2), 2011, $\quad$ pp. 220238.

19. Luo Q and Mao X. Stochastic population dynamics under regime switching. J. Math. Anal. Appl.334, 2007, pp. 69-84.

20. Michael Steel J. Stochastic calculus and financial applications. Springer-Verlag. 2003.

21. Naresh R, and Omar S. An epidemic model for the transmission dynamics of HIV/AIDS and another infection. International Journal of Mathematical Archive-1(3), 2010, pp. 68-72.

22. Oksendal-B Stochastic Differential Equations an Introduction with Applications Fifth Edition; corrected printing springer verlag Heidelberg New york . 2000. pp. 1-352.

23. P. LaSalle and S. Lefschetz, Stability by Liapunov's direct method. Academic Press. 1961.

24. Perto. L. Differential Equations and Dynamical Systems. 2nd edition. Springer, New York. 1996.

25. Ray Waston. A useful Random Time-Seal Transformation for The Standard Epidemic Model. J. Appl. Prob.17, 1980, pp. 324-332.

26. Ray Waston. On the Size Distribution for Some Epidemic Models. J. Appl. Prob.17, 1980, pp. 912-921.

27. Robert N and May. Population Biology of infectious diseases I. International centre of theoritical physics, 1982, pp. 1-9.

28. Ruoyan Sun. Global stability of the endemic equilibrium of multi group SIR models with nonlinear incidence. Computers and Mathematics with Applications 60, 2010, pp. 2286-2291.

29. Sallet. Modélisation et simulation en épidémiologie.R0_EPICASA09.INRIA \& IRD. 2010, pp 1-38. http://www.math.univ-metz.fr/ sallet/R0_EPICASA09.pdf

30. Sudipa Chauhan, Om Parakash Misra, Joydip Dhar. Stabiliy Analysis of SIR model with vaccination. American journal of computational and applied mathematics, 4(1), 2014, pp. 17-23. 
31. Takeuchi and W. Ma. Stability analysis on a delayed SIR epidemic model with density dependent birth process, Dy-nam. Contin. Discrete Impuls. Systems, 5, 1999, pp.171-184.

32. Volodymyr Makarov, Denis Dragunov. A numeric-analytical method for solving the Cauchy problem for ordinary differential equations. Applied Mathematics and Computation, 2010, pp. 1-26.

33. W. Ma, Y. Takeuchi, T. Hara and E. Beretta, Permanence of are SIR epidemic model with distributed time delays. Tohoku Math. J. 54, 2002, pp. 581-591.

34. W. Wang, Global behavior of an SEIR epidemic model with time delay. Appl. Math. Letters.15, 2002, pp. 423428.

35. Wen L and Yang X. Global stability of a delayed SIRS model with temporary immunity. Chaos, Solitons and Fractals 38, 2008, pp. 221-226.

36. Xiao, L Chen and F. Ven den Bosch, Dynamical behavior for a stage-structured SIR infectious disease model. Nonlinear Anal. Real World Appl 3, 2002, pp.175-190.

37. Z. Ma, J. Liu and J. Li, Stability analysis for differential infectivity epidemic models. Nonlinear Anal. Real World Appl 4, 2003, pp. 841-856.

38. Zhang F and Zhen Li and Zhang F. Global stability of an SIR epidemic model with constant infectious period. Applied Mathematics and Computation 199, 2008, pp. 285-291. 